\title{
Complete Pelvic Mass Regression Following Tuberculosis Treatment as Indicated by F18-FDG PET/CT
}

\author{
Ece Akbulut ${ }^{1}$, Mehmet Tarik Tatoğlu² (D), Ferhat Arslan ${ }^{1}$ \\ 1 Department of Infectious Diseases and Clinical Microbiology, Medeniyet University Göztepe Training and Research \\ Hospital Ringgold Sandard Institution, İstanbul, Turkey \\ 2 Department of Nuclear Medicine, Medeniyet University Göztepe Training and Research Hospital Ringgold Sandard \\ Institution, İstanbul, Turkey
}

\begin{abstract}
A 19-year-old woman with a history of tuberculosis (TB) in her family presented to the infectious disease clinic of our hospital with right flank pain, nausea, vomiting, a 10$\mathrm{kg}$ weight loss, and decreased urine volume for the past two months. Empirical anti-TB treatment was initiated due to her family history of tuberculosis, a positive purified protein derivative skin test, and the presence of a nodule in the posterobasal segment of the lung upon F18-FDG PET/CT. We aimed to present the complete regression of a pelvic mass lesion after empirical tuberculosis treatment that was visualized on follow-up F18-FDG PET/CT imaging and discuss its contribution to the literature. F18-FDG PET/CT imaging followup may offer an invaluable resource for monitoring extrapulmonary involvement of TBinfected patients. The F18-FDG PET/CT imaging method may also contribute to the followup of granulomatous and inflammatory diseases.

Keywords: Tuberculosis, F18-FDG PET/CT, infectious disease
\end{abstract}

\section{INTRODUCTION}

A 19-year-old woman with a history of tuberculosis (TB) in her family presented to the infectious disease clinic of our hospital with right flank pain, nausea, vomiting, a 10-kg weight loss, and decreased urine volume for the past two months. Approximately five years ago, within a year of family TB contact, the patient had been admitted to another hospital with chronic painful left submandibular lymph node enlargement. A histopathological examination had revealed chronic active inflammation in the fat and connective tissues, including polyclonal plasma cells. After removing the lymph node, no further treatment was sought due to the self-limited nature of the clinic. The patient had been well until approximately two months before her presentation. Neither latent TB screening nor TB treatment had been implemented in recent years. The physical examination revealed a cachectic appearance, a blood pressure of 110/70
Corresponding Author: Ferhat Arslan

E-mail:

ferhatarslandr@gmail.com

Received: October 22, 2020

Accepted: December 21, 2020

Published: December 31, 2020

Suggested citation:

Akbulut E, Tatoğlu MT, Arslan F.

Complete Pelvic Mass Regression Following Tuberculosis

Treatment as Indicated by F18-FDG PET/CT. Infect Dis Clin Microbiol 2020; 3: 175-178.

DOI: $10.36519 / \mathrm{idcm} .2020 .0025$ 

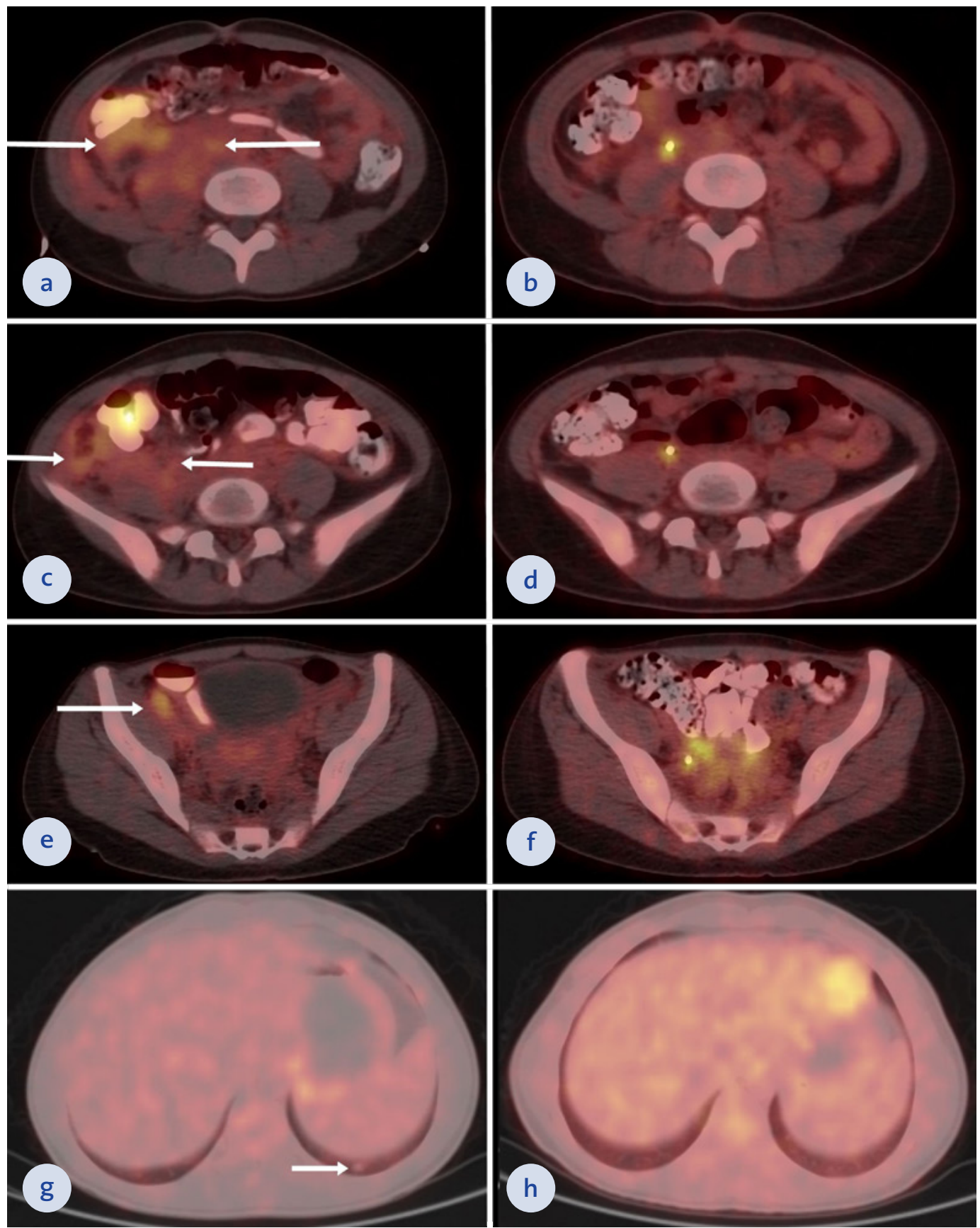

Figure 1. Pre-treatment axial F18-FDG PET/CT fusion images and axial CT images extending from the anteroinferior aspect of the right kidney to the paracaval area and the anterior of the right psoas major muscle to the right iliac fossa reveal an invaded right lateral peritoneal surface and an irregularly contoured, heterogeneous, slightly hypermetabolic soft tissue lesion and right external iliac lymph node (SUVmax: 3.7) (a, c, e).

Right external iliac lymph node and the soft tissue lesion that was interpreted as the involvement of urinary tuberculosis showed

almost complete metabolic and morphological regression in the post-treatment axial F18-FDG PET/CT fusion images and axial $C T$ images (b, $d, f)$.

Pre-treatment axial F18-FDG PET/CT fusion images and axial $C T$ images also revealed a 7-mm ametabolic nodule in the lower lobe posterior basal segment of the left lung $(\mathrm{g})$. No lesion was seen in the post-treatment axial F18-FDG PET/CT fusion images and axial CT images (h) 
$\mathrm{mm} \mathrm{Hg}$, and right pelvic tenderness. The remainder of the examination was normal. A laboratory evaluation produced the following results: erythrocyte sedimentation rate (ESR): $102 \mathrm{~mm} / \mathrm{h}$, C-reactive protein (CRP): $7.08 \mathrm{mg} / \mathrm{dl}(0-0.5 \mathrm{mg} / \mathrm{dl})$, hemoglobin(hg): $8.8 \mathrm{~g} / \mathrm{dl}$, and mean corpuscular volume: 67.2 fl. A human immunodeficiency virus (HIV) antibody test was negative. A purified protein derivative (PPD) skin test was recorded as $16 \mathrm{~mm}$. Pyuria was detected upon urinalysis, but a urine culture revealed no growth. A computed tomographic (CT) scan of the abdomen was notable for a $70 \times 30 \mathrm{~mm}$ soft tissue density mass invading the right psoas muscle and nearby fat tissue that could not be distinguished from the surrounding intestinal segments. Both the urine and a fine-needle aspirate of the pelvic tissue produced no TB or any bacteria growth. Ehrlich-Ziehl-Neelsen staining and mycobacterium polymerase chain reaction also remained negative. Empirical anti-TB treatment (300mg isoniazid, 600mg rifampin, $1250 \mathrm{mg}$ pyrazinamide, and 700mg streptomycin) was initiated because of TB history of the family, the positive PPD skin test, and a nodule in the posterobasal segment of the lung in F18-FDG positron emission tomography/computed tomography (PET/CT) (Figure 1g). During the fourth month of treatment, the ESR was $3 \mathrm{~mm} / \mathrm{h}$, the CRP had decreased to $<0.2 \mathrm{mg} / \mathrm{dl}$, and the hg rose to $12.1 \mathrm{~g} / \mathrm{dl}$. The patient fully recovered. A follow-up F18-FDG PET/CT examination after one year of therapy showed decreased uptake in the patient's pelvic and lung involvements and a resolution of the inflammation (Figures $1 \mathrm{~b}, \mathrm{~d}, \mathrm{f}, \mathrm{h}$ ).

\section{DISCUSSION}

Mycobacterium tuberculosis infection can be classified as an active or latent disease process depending on the bacterial and individual genetic and immunological factors involved (1). Prior research has shown that inflammatory markers (interferon-gamma) and imaging techniques (F18-FDG PET/ $\mathrm{CT}$ ) may reveal early events that determine the course of the disease (2). F18-FDG PET/CT clinical studies are typically limited to lung and lymph node infections, but tuberculosis can occur in any tissue in the body, making it a great imitator of other diseases (3). This case illustrated the contribution of PET/CT in assessing the follow-up treatment response in probable extrapulmonary TB. F18-FDG PET/CT imaging follow-up may offer an invaluable contribution, especially in some extrapulmonary involvements that cannot be followed using routine microbiological assessments and also in HIV co-infected patients, cancer patients, or those with multidrug resistance (4-6). Clinicians should still follow standard TB guidelines for treatment modalities and duration of therapy based on bacterial resistance and regions of involvement $(7,8)$. However, because F18-FDG PET/CT can reveal early inflammatory changes in TB's pathogenesis, it may be a good surrogate marker to facilitate better clinical decisions in specific situations.

Pre-treatment axial F18-FDG PET/CT fusion images and axial CT images extending from the anteroinferior aspect of the right kidney to the paracaval area and the anterior of the right psoas major muscle to the right iliac fossa reveal an invaded right lateral peritoneal surface and an irregularly contoured, heterogeneous, slightly hypermetabolic soft tissue lesion and right external iliac lymph node (SUVmax: 3.7) (a, c, e).

Right external iliac lymph node and the soft tissue lesion that was interpreted as the involvement of urinary tuberculosis showed almost complete metabolic and morphological regression in the post-treatment axial F18-FDG PET/CT fusion images and axial CT images ( $b, d, f)$.

Pre-treatment axial F18-FDG PET/CT fusion images and axial CT images also revealed a 7-mm ametabolic nodule in the lower lobe posterior basal segment of the left lung (g). No lesion was seen in the post-treatment axial F18-FDG PET/CT fusion images and axial CT images (h). 
Informed Consent: Written consent was obtained from the patient.

Peer-review: Externally peer-reviewed

Author Contributions: Concept - M.T.T.; Design - F.A.; Supervision - M.T.T;; Fundings - F.A.; Materials - F.A.; Data Collection and/or Processing - E.A.; Analysis and/or Interpretation - M.T.T.; Literature Review - E.A.; Writer -E.A.; Critical Reviews - F.A.
Conflict of Interest: The authors have no conflict of interest to declare.

Financial Disclosure: The authors declared that this study has received no financial support

\section{REFERENCES}

1 Zumla A, Raviglione M, Hafner R, von Reyn CF. Tuberculosis. N Engl J Med 2013; 368: 745-55.

2 Coleman MT, Maiello P, Tomko J, Frye LJ, Fillmore D, Janssen C, et al. Early changes by 18Fluorodeoxyglucose positron emis sion tomography coregistered with computed tomography predict outcome after Mycobacterium tuberculosis infection in cynomolgus macaques. Infect Immun 2014; 82: 2400-4.

3 Ganchua SKC, Cadena AM, Maiello P, Gideon HP, Myers AJ, Junecko BF, et al. Lymph nodes are sites of prolonged bacterial persistence during Mycobacterium tuberculosis infection in macaques. PLoS Pathog 2018; 14: e1007337.

4 Dureja S, Sen IB, Acharya S. Potential role of F18 FDG PET-CT as an imaging biomarker for the noninvasive evaluation in uncomplicated skeletal tuberculosis: a prospective clinical observational study. Eur Spine J 2014; 23: 2449-54.

5 Shejul Y, Chhajed PN, Basu S. 18F-FDG PET and PET/CT in diagnosis and treatment monitoring of pyrexia of unknown origin due to tuberculosis with prominent hepatosplenic involvement. J Nucl Med Technol. 2014; 42: 235-7.

6 Malherbe ST, Shenai S, Ronacher K, Loxton AG, Dolganov G, Kriel M, et al. Persisting positron emission tomography lesion activity and Mycobacterium tuberculosis mRNA after tuberculosis cure. Nat Med 2016; 22: 1094-100.

7 Nahid P, Dorman SE, Alipanah N, Barry PM, Brozek JL, Cattamanchi A, et al. Executive summary: Official American Thoracic Society/Centers for Disease Control and Prevention/ Infectious Diseases Society of America clinical practice guidelines: treatment of drug-susceptible tuberculosis. Clin Infect Dis 2016; 1; 63: 853-67.

8 Rabahi MF, Rodrigues da Silva JL, Ferreira ACG, Tannus-Silva DGS, Conde MB. Tuberculosis treatment. J Bras Pneumol 2017; 43: 472-86. 